\title{
¿SÓLO VAN A TRABAJAR SI RECIBEN LOS FONDOS? Management, regulación moral y escuelas en la periferia metropolitana (Córdoba, Argentina)
}

\author{
Maria Cecilia Bocchio 58 \\ Silvia Mariela Grinberg 59
}

\section{RESUMEN}

Las políticas educativas en la era del management asociadas a la "Nueva Gestión Pública" (NGP) vienen ejerciendo, desde la década del 90 , claros efectos en la vida de las escuelas que, en el contexto argentino, adquieren dinámicas, formas y efectos específicos que regulan la moral y las prácticas de los directivos, apelando al componente ético de su trabajo. Este artículo se enmarca en una investigación que analiza el trabajo del director escolar y los procesos de regulación de las políticas educativas orientadas a garantizar la escolaridad secundaria. Presentamos un estudio de caso donde abordamos el trabajo del director de una escuela localizada en las periferias de la Ciudad de Córdoba a la que asisten alumnos sumidos en crecientes procesos de pauperización social. A modo de hipótesis proponemos que el llamado al compromiso para con el alumnado y sus demandas sociales insatisfechas se transforman en el motor que regula moralmente al director y lo obliga a trabajar para y a pesar de no recibir los prometidos fondos que los proyectos ministeriales deben proveer.

Palabras clave: Dirección Escolar. Management. Regulación Moral.

\section{SÓ IRÃO TRABALHAR SE RECEBEREM O FINANCIAMENTO? \\ Management, regulação moral e escolas da periferia metropolitana}

(Córdoba, Argentina)

\section{RESUMO}

As políticas educativas na era do management, associadas à "Nova Gestão Pública" (NGP), vêm exercendo desde a década dos anos 90, efeitos claros na vida das escolas que, no contexto argentino, adquirem dinâmicas, formas e efeitos

58 Doctorado en Política y Administración Educativa. Universidad de Lisboa. Universidad Nacional de Córdoba, Argentina. E-mail: mcbocchio@gmail.com.

59 Investigadora del CONICET. Directora del Centro de Estudios en Pedagogías Contemporáneas (CEPEC) y Profesora regular de Sociología de la Educación y Pedagogía, EHU-UNSAM. Profesora titular de Pedagogía y Coordinadora del Área Sociopedagógica de la UNPA-UACO. E-mail: grinberg.silvia@gmail.com. 
específicos que regulam a moral e as práticas dos gestores, apelando ao componente ético do seu trabalho. Este artigo enquadra-se numa investigação que analisa o trabalho do diretor escolar e os processos de regulação das políticas educativas orientadas a garantir a escolaridade secundária. Apresentamos um estudo de caso onde se aborda o trabalho do diretor de uma escola situada na periferia da cidade de Córdoba, onde assistem alunos evadidos em processos crescentes de pauperização social. Hipotetizamos que o compromiso com os alunos e as suas demandas sociais insatisfeitas transformam-se no motor que regula moralmente ao diretor e obliga ele a trabalhar, mesmo sem receber o financiamento para os projetos que o Ministério deve fornecer.

Palavras-chave: Diretor Escolar. Management. Regulação Moral.

\title{
WILL YOU ONLY WORK IF YOU GET THE FUNDS?
}

\section{Management, moral regulation and schools in the metropolitan periphery (Córdoba, Argentina)}

\begin{abstract}
In Argentina, since the 1990s, management logic has been the guiding principle in educational policy; it affects daily life at school and the work of principals. In this article, we propose that the logic associated with New Public Management (NPM) encourages moral regulation of practices, which has particular ethical implications for how principals perform their work. This article is part of broader research on regulatory processes in educational policies aimed at ensuring compulsory secondary schooling and processes we address from the perspective of principals attempting to do their work. We present a case study of a principal at a school located on the outskirts of the city of Córdoba. The students who attend the school are inserted in social processes of pauperization. Within the framework of governmentality studies in education and on the basis of empirical research performed at that school, we will discuss some of the aspects that the regulation of school life in management times entails. As a hypothesis we propose that the call to commitment with students and their unsatisfied social demands become the motor that regulates morally the principal and forces him to work for and despite not receiving the promised funds that the ministerial projects must provide.
\end{abstract}

Keywords: School Principal. Management. Moral Regulation.

\section{INTRODUCCIÓN}

¿Señor director, considera que va a trabajar sólo si recibe los fondos económicos del proyecto? Es la pregunta que recibe a modo de respuesta el director de una escuela secundaria por parte de un funcionario del ministerio de educación responsable de un conjunto de programas 
orientados a la mejora de los niveles de retención y promoción escolar. Ello mientras el directivo reclama al funcionario una respuesta respecto de cuándo iban a recibir los fondos a los que habían aplicado y así poder planificar qué iban a hacer y saber qué cantidad de dinero iban a disponer. Lejos de cualquier juicio que desde ya, es posible realizar a esta pregunta, sin duda, retórica el interrogante que orienta el presente artículo gira en torno a las condiciones de emergencia de ese enunciado así como acerca de sus efectos. Nos cuestionamos qué es lo que hace posible que este diálogo haya ocurrido y que el directivo que lo recibe como respuesta se haya visto afectado por ese enunciado. Nos referimos al suelo arqueológico de esa enunciación (DELEUZE, 2014; FOUCAULT, 1972), a las dinámicas de la vida escolar que se producen en ese suelo así como a los efectos de las tecnologías y regulaciones en esas dinámicas.

En este marco, en el presente artículo nos proponemos debatir, a través de resultados de una investigación empírica realizada en escuelas secundaria, sobre algunos de los aspectos que presenta la regulación de la vida escolar en tiempos gerenciales. Al respecto proponemos que las lógicas propias de la gestión se ensamblan de un particular modo en la vida escolar a través de la acción directa de quienes allí trabajan, transformando cuestiones como contar con cargos docentes para hacer frente a nuevas demandas (en algunos casos provenientes de nuevas legislaciones) en una cuestión de predisposición personal para el trabajo. Las narrativas de la gestión conforman ese suelo arqueológico para la apelación moral que trae consigo y se constituye en oxímoron de la vida escolar. Esto es, el mismo programa que convoca a las escuelas a formular proyectos reclama moralmente al director que trabaje a pesar de no recibir los recursos económicos. A través de resultados de investigación discutimos elementos para la comprensión de los modos que presenta la regulación de la vida escolar en tiempos gerenciales, que asentada en las retóricas de la participación y el compromiso delega a los sujetos la responsabilidad moral sobre la actuación de las políticas en las escuelas. Entendemos que la vida 
escolar articulada en torno a hilos propios de la autoayuda y una particular invocación afectiva y moral, se vuelve una carrera de postas, muchas veces, cruel para las instituciones (AUTOR 2015; BERLANT, 2011). Esto adquiere especiales ribetes en escuelas emplazadas en contextos de pobreza urbana llamadas a la cooperación y autogestión como modos de resolver la escasez de recursos en la que se encuentran.

Como lo proponemos más adelante no es la cooperación aquello que se pone en cuestión sino más bien hacer eje en esa lógica de la gestión comprometida como un modo de resolver los problemas de la comunidad. Más aún cuando, justamente, no es la ausencia de esas prácticas sino su permanente presencia aquello que permite a escuela y comunidad hacerse y auto hacerse (AUTOR, 2011). Es desde esta perspectiva que proponemos que la "Nueva Gestión Pública" (NGP) se vuelve regulación moral de la vida escolar dejando a las escuelas sobrecargadas de responsabilidades sin los recursos para hacerles frente, en un particular estado que combina agobio y empuje permanente.

América Latina en general y Argentina en particular está impregnada por las dinámicas de la gerencia de las políticas públicas (AUTOR, 2008) que se desarrollan en la lógica de la NGP. Como lo han señalado investigadores de países americanos (POPKEWITZ, 1996; APPLE, 2001; BARRIGA \& ESPINOSA, 2001); europeos (MAROY \& DUPRIEZ, 2000; BARROSO, 1997; BALL \& VAN ZANTEN, 1998; VIÑAO FRAGO, 2002) y oceánicos Whitty, Power \& Halpin (1998) las políticas educativas promovidas desde la década del 80 , han tenido una clara influencia en la transformación de los modos de regulación de los sistemas educativos, en el trabajo del director escolar y puntualmente en la promoción de instrumentos de gestión empresarial como: la gestión por proyecto, los planes de mejora institucional, las buenas prácticas, las evaluaciones estandarizadas, entre otras acciones que proponiendo tornar más eficiente la escuela estatal, pasaron a formar parte del trabajo cotidiano de directivos y docentes. 
En este trabajo, nos preocupa analizar las dinámicas y los efectos que ciertos instrumentos y prácticas de la NGP promueven en el director escolar y en las dinámicas cotidianas de la escolaridad. La noción de efectos (BALL, 2002) y afectos (BERLANT, 2011; AHMED, 2010) nos importa especialmente en tanto nos permite dar cuenta de esa regulación, tal como se produce en la vida institucional.

En este marco, resulta necesario señalar que este artículo y su título nos remonta a una conversación que mantuvimos con el director de una de las escuelas secundarias donde estamos llevando a cabo el trabajo de campo de una investigación en curso, centrada en el análisis de las políticas orientadas a garantizar la escolaridad secundaria obligatoria60. En el diálogo, el director relata una situación que vivió con un funcionario jerárquico del Ministerio de Educación de la provincia de Córdoba, en ocasión de una reunión con directivos donde se presentaba un nuevo proyecto educativo orientado a elevar los niveles de promoción escolar. El proyecto iba a ser financiado por el gobierno y si bien aún no había fecha para la recepción de los fondos económicos que se iban a otorgar, el funcionario se ocupó (en primer lugar) de explicarles cómo debían rendir cuentas de los recursos económicos provistos, o, más bien que iban a ser provistos en algún momento, sin especificar cuándo. Ante esta situación el interrogante planteado por algunos directivos fue: ¿̇Cómo vamos a rendir algo sin recibir los fondos, sin saber todavía qué vamos a hacer? La respuesta recibida fue un nuevo interrogante: ¿̇Consideran que van a trabajar sólo si reciben los fondos económicos del proyecto?

La estancia de investigación en la escuela, la vivencia del día a día del trabajo del equipo directivo y de los docentes nos permite plantear como hipótesis que el trabajo de dirigir "exitosamente" una escuela pública se

\footnotetext{
60 En Argentina a lo largo de los últimos veinte años se produjeron dos reformas estructurales del Sistema Educativo. La primera fue la Ley Federal de Educación № 24.195 en el año 1993 y la segunda, más reciente y vigente, en el año 2006, denominada Ley de Educación Nacional (LEN) N² 26.206. Esta última legislación regula la extensión de la obligatoriedad de la escuela secundaria hasta su culminación y propone una escolaridad obligatoria de 13 años en todo el país y desde los 4 años.
} 
presenta como una batería de principios morales que performan el trabajo del director orientando su accionar. Principios que se adicionan (MAROY, 2011) en la lógica de la gestión escolar eficiente y estratégica. En otras palabras, la puesta en acto (BALL ET AL, 2012) de los proyectos educativos se vuelve un problema de "gestión estratégica" (aunque el ministerio sea quien promueve las políticas educativas), donde el director es llamado a producirse en su self y a transmitir esa filosofía a sus docentes.

La pregunta retórica del funcionario también coloca en el centro de la organización escolar al trabajo del director escolar a quien le compete la ejecución diversas políticas educativas con o sin los recursos necesarios, valga la redundancia, para tal ejecución. Se demandan competencias necesarias para ejecutar programas y proyectos educativos que atiendan a las necesidades de una matrícula escolar en creciente proceso de expansión y pauperización. Así, los problemas, desigualdades e injusticias de la vida escolar se transforman en una cuestión de gestión o, más bien, de buena o mala gestión y/o de modelos tradicionales que deben superarse para atender a las nuevas necesidades sociales. Si un alumno no aprende, o no aprende tal como se espera que lo haga, la cuestión radica en cómo se gestiona el aprendizaje. Si los alumnos abandonan la escuela se trata de un directivo o de un cuerpo de docentes que no monitorea el proceso de aprendizaje, no desarrolla estrategias de retención y, en cuanto al primero, que no ha logrado volverse un verdadero líder, motor de los procesos de cambio que deben promoverse. La desigualdad educativa se ha vuelto una cuestión de gestión y ésta es la "llave del cambio o la llave mágica" que debe aplicarse para superar cada conflicto (AUTOR, 2008).

En este artículo nos interesa específicamente a partir de un estudio en caso analizar la performatividad de estos enunciados. Esta mirada ocurre en línea con otras investigaciones (BALL, 2002; EACOT, 2011; LUENGO NAVAS \& SAURA CASANOVA, 2012; ROCKWELL, 2013; SOTO LAGOS ET AL, 2016) que abordan los efectos de estas políticas a nivel de las instituciones y de los sujetos que actúan las políticas. Además nos basamos en un conjunto de 
estudios que venimos desarrollando los cuales nos posibilitan describir los efectos y la afección que las políticas y sus instrumentos de gerenciamiento están ejerciendo en el trabajo del director, performándolo.

A continuación, en primer lugar, recuperamos algunas de las particularidades que la racionalidad del management adquiere en el campo educativo; seguidamente presentamos el enfoque metodológico adoptado atendiendo el contexto y los sujetos implicados en el trabajo en terreno. En tercer lugar caracterizamos el trabajo del director de escuela procurando diferenciar las tareas que es llamado a gestionar. Finalmente desarrollamos algunos apuntes conclusivos sobre el trabajo que desempeña este director que es considerado por sus colegas y superiores como exitoso.

\section{LA RACIONALIDAD DEL MANAGEMENT EN LAS ESCUELAS}

La NGP emerge ya hace décadas como la fórmula para responder a las evaluaciones de crisis del Estado, propiciando la delegación de responsabilidades en lo local para actuar las políticas públicas (CLARKE \& NEWMAN, 1997; HOOD, 1991). Concretamente en educación aparece como "gestión centrada en la escuela", y se focaliza en la promoción de márgenes de autonomía en las instituciones para responder a las demandas específicas de alumnado (BARROSO, 2005). La gestión se legitima en oposición a la "antigua" administración, entendida como la disposición de los medios para conseguir un fin, propiciando que las estructuras trabajen para alcanzar un objetivo dado. La escuela libre de las ataduras de la vieja burocracia parece ganar en capacidad para dar respuestas a las demandas de su entorno. Por tanto, la gestión va un paso más allá: se incorpora a los medios como objeto de la planificación-previsión. Esto significa que se trabaja sobre el proceso, dado que gestionar no sólo implica disponer los medios, sino también crearlos y articularlos (Autor, 2006). La buena gestión aparece, así, como la herramienta principal para obtener recursos, tornar eficiente el funcionamiento de la organización escolar y la 
toma de decisiones en materia educativa. A fin a una perspectiva gerencial Albariño (2000) lo define de este modo:

La gestión centrada en la escuela, no es un fin en sí mismo (...) es una herramienta que potencialmente tendría sus fortalezas en comprometer la participación de los interesados (stake holders) en el proceso (con mayor fuerza de lo que se observa en el tradicional esquema top-down arriba/abajo de administración) y lograr así satisfacer de mejor manera las necesidades de los alumnos (p.19).

La respuesta a las demandas del alumnado acaban siendo resueltas en función de las condiciones construidas y de los recursos adquiridos por los sujetos en las escuelas. La inversión del esquema top-down se basa en el compromiso y es desde allí que propone delegar a las escuelas la responsabilidad sobre los procesos escolares. De hecho es ese compromiso, el "pivote central" de la lógica gerencial, aquello que aparece como reclamo en la pregunta que da origen al título del presente artículo.

Para Ball (2013) las políticas educativas diseminadas desde este nuevo paradigma sobre lo público que define Albariño, reflejan un "ensamble genérico de políticas internacionales"61, cuya construcción se basa en un conjunto de tecnologías políticas comunes. Estas tecnologías responden a un "modelo único para todos"62 y se fundamentan en un particular modo de comprender la modernización y transformación del sistema y de la organización del sector público. Diferencia tres tecnologías: market form, management, performativity63. Ahora bien, estas tecnologías ocurren de un

\footnotetext{
${ }^{61}$ En inglés "generic global policy ensemble"

62 En inglés "one-size-fits-all"

63 Parafraseamos a Ball (2013) quien caracteriza estas tecnologías del siguiente modo: 1. "Market form", la creación de mercados educativos se asienta en la introducción de dinámicas de competición en el sistema público, de este modo se construye un medio ambiente basado en la competividad de los proveedores de los servicios educativos, tales como escuelas, universidades, institutos. 2. "Management" la figura central para la reorganización del sector público es el manager o el director escolar, quien se transforma en un agente de cambio clave para la política educativa. Como parte de los imperativos de las reformas del sector público el management representa la contracara de la burocracia y el profesionalismo. 3. "Performativity" definida como una cultura o un "sistema de terror", constituye un régimen para la rendición de cuentas que supone comparación, juzgamiento y el despliegue de mecanismos de control y cambio. Así, profesores y directivos son objeto
} 
modo muy particular en el caso que nos ocupa. A modo de ejemplo, en Argentina no se han puesto en marcha procedimientos ni normativas que involucren la competencia entre escuelas en rankings escolares o bien por la obtención de fondos económicos. Sin embargo, el gerenciamiento y la performación de sus sujetos atraviesan la vida escolar.

Según Eacott (2011) la planificación estratégica basada en la escuela es un mecanismo para poner en acto políticas neoliberales. Es en esa línea de análisis que Barroso (2005) remite a la post-burocracia como un nuevo modo de regulación de la acción estatal, que cobra hegemonía entre los años 60 y 90 e involucra a los organismos internacionales de crédito como garantes para el cambio de la imagen ineficiente del Estado. En la organización escolar esos cambios proponen reestructurar el servicio público asignando un espacio central a lo local para ejecutar las políticas procurando:

La sustitución del control jerárquico por el auto-control, la flexibilización de las estructuras con la sustitución de la organizaciones piramidales por organizaciones en red y el fin de la separación entre organización formal e informal, otorgando particular importancia a las relaciones personales y la afectividad (BARROSO, 2005, p. 430).

Las modalidades pos-burocráticas de regulación del gobierno de la educación para Carvalho (2016) complejizan este proceso, propiciando nuevos instrumentos como contratos, evaluaciones, proyectos, planes de mejora, buenas prácticas. En conjunto, estos instrumentos promueven una regulación que combina, por un lado, la implicación voluntaria de los actores con la monitorización de sus desempeños.

En el marco de estos debates entendemos que el gobierno de los otros (FOUCAULT, 2006, 1999) ocurre a través del trabajo del director y esto demanda analizar cómo en tiempos de gerenciamiento se reconfigura la dirección escolar, en el cotidiano del hacer escuela. Consideramos que el

de evaluación y se enfrentan al cambio en los valores que orientan sus labores cotidianas. 
gerenciamiento se ha vuelto la racionalidad de nuestra época y se presenta como modos de auto-hacerse en las sociedades contemporáneas. Donde la gestión de sí opera como mecanismo y modo de manifestación de la verdad de nuestra época. De manera que lejos de las hipótesis que refieren a la ausencia del gobierno y /o del Estado, nos encontramos con maneras de conducción de la conducta donde el gobierno de los otros deviene como pliegue del gobierno de sí (AUTOR, 2015, p.13).

En el caso de las políticas educativas se promueve el empoderamiento del director escolar, dotándolo de autonomía, deviniendo en sujeto y objeto de sí, en otras palabras promoviendo la auto-gestión para conducir una escuela, atendiendo a múltiples demandas sociales y educativas. En este contexto, se propician las condiciones para que opere lo que Rose (1996, p.122) denomina "el gobierno a través de la comunidad", una tecnología que implica una variedad de estrategias para inventar e instrumentalizar estas dimensiones de lealtad entre los individuos y las comunidades al servicio de proyectos de regulación, reforma o movilización social. Siguiendo el planteo del autor, a la ética de los sujetos se le concede una nueva relación activa con su estado, en términos de sus estrategias y capacidades para la gestión de sí. En caso contrario supone un rehúso a la comunidad y a la auto-responsabilidad que su labor le demanda, dando cuenta-ademásde ciertos niveles de ineptitud.

En suma, nos encontramos ante tecnologías como el management y la performatividad (Ball); las relaciones interpersonales y la afectividad (Barroso); la gestión de sí (Autor), el gobierno a través de la comunidad y la nueva ética (Rose) que están afectando de manera notable la regulación del trabajo del director y promoviendo la reconfiguración del ser-directivo. Sobre esas lógicas, a continuación nos ocupamos. 


\section{LA INVESTIGACIÓN: BREVES APUNTES METODOLÓGICOS Y CONTEXTUALES}

El trabajo de investigación se desarrolló desde una perspectiva cualitativa con el objeto de describir las dinámicas que atraviesan a la vida escolar especialmente desde el punto de pista del directivo, en escuelas secundarias emplazadas en las periferias de la ciudad de Córdoba (Argentina), cuyo alumnado está inserto en crecientes procesos de pauperización social. Desarrollamos un estudio en caso y recurrimos a elementos del enfoque etnográfico para observar y describir el cotidiano escolar y los espacios institucionales (ROCKWELL, 2011).

El trabajo que aquí discutimos se desarrolla desde 2015, en una escuela secundaria de la ciudad de Córdoba64, emplazada en una "Ciudad-Barrio" construida para dar respuesta a un proceso de re-localización de asentamientos -comúnmente llamados en la región como villas miseria o favelas-; es decir, grupos de viviendas caracterizadas por la extrema precariedad y radicadas en zonas afectadas recurrentemente por fenómenos climáticos como las inundaciones. La construcción de CiudadesBarrios responde a una política habitacional financiada con un crédito del Banco Interamericano de Desarrollo (BID)65. Puntualmente la Ciudad-Barrio donde se desarrolla esta investigación se sitúa en las periferias de la ciudad y fue inaugurada en el mes de octubre del año 2005 para relocalizar siete asentamientos precarios. En la misma se construyeron 359 viviendas, una escuela secundaria (donde estamos desarrollando el trabajo de campo), una escuela primaria, un jardín de infantes y un centro de atención primaria de la salud.

En este artículo recuperamos observaciones, conversaciones informales, entrevistas en profundidad desarrolladas con el director escolar durante el ciclo lectivo 2015 y a comienzo de 2016. Los ejes que guiaron el

\footnotetext{
${ }^{64}$ La ciudad de Córdoba es la segunda ciudad más populosa de Argentina después de la ciudadd de Buenos Aires.

${ }^{65}$ Esta política consistió en la construcción de 12.000 unidades habitacionales, agrupadas en 14 "barrios-ciudades" que albergan a 7.479 familias.
} 
desarrollo de las entrevistas se vinculan con: la puesta en acto (BALL ET AL., 2012) de las políticas socio-educativas, la coordinación del trabajo con el cuerpo docente, los modos de vincularse con las familias y con el entorno escolar. Cabe señalar que esta escuela suele ser vista por docentes, directivos y funcionarios del ministerio como una escuela que "funciona bien" o una escuela exitosa. Si bien esta investigación no se focaliza en analizar las causas de ese éxito, el desarrollo del trabajo nos permitió comprender algunos de los elementos sobre los que éste descansa y los e/afectos que el trabajo de ser exitoso produce en el director y en los docentes.

\section{ACERCA DE LA ESCUELA Y EL BARRIO}

La escuela fue trasladada de edificio y relocalizada a $7 \mathrm{~km}$ de su emplazamiento original. La re-inauguración fue en octubre de 2005. Así, describe el actual director las transformaciones vividas:

En octubre de 2005 se habían cambiado de edificio, perdimos muchos de los alumnos. En 2006 hubo sólo seis egresados, este año tenemos 30 y pico. Es un proceso, esperemos que en diez años tengamos 50 o 60 (SEGUNDA ENTREVISTA DIRECTOR, 2015).

Luego de 9 años, la escuela posee aproximadamente 385 alumnos, cuenta con un equipo directivo, docente y preceptores con relativa estabilidad en el tiempo, y un gabinete psicopedagógico. Al respecto, cabe aclarar que en Argentina los profesores de escuela secundaria generalmente no concentran horas en una sola escuela. Por ello, suelen trabajar en promedio en 3 o 4 instituciones por lo que una parte importante de su horario de trabajo (no pago) implica movilizarse entre las instituciones. Es por ello que sin eufemismos suelen recibir el nombre de profesor taxi.

Como veremos más adelante, esto no constituye un dato menor para la gestión cotidiana de la escuela y de los equipos de docentes que al conseguir cierta concentración horaria han generado mejores condiciones de trabajo docente para que esta escuela pueda progresivamente elevar 
los niveles de retención y promoción escolar, en el marco de un edificio escolar nuevo y en buen estado de mantenimiento. De este modo lo resume el director:

En líneas generales en 2015 retuvimos el $90 \%$ de los chicos. Ahora tenemos dos quinto años con muchos alumnos y eso es muy positivo, en quinto de la tarde tenemos 27 el año pasado eran 14, o sea mejoramos mucho (TERCERA ENTREVISTA DIRECTOR, AÑO 2016).

Tal como lo señala el director, los buenos resultados los han transformado en una escuela "poco problemática" que cuenta con el apoyo de la inspectora escolar. Una escuela que recibe alumnos provenientes de familias que poseen un nivel socio- económico bajo, caracterizadas por ser (en un gran porcentaje) familias monoparentales, generalmente sostenidas por madres beneficiarias de planes sociales. La escuela en palabras del director consigue incluir y retener al alumno propiciando condiciones para el egreso (en la mayoría de los casos) de la primera generación familiar en concluir la escuela secundaria. Nociones como "mejoramos mucho" o "conseguimos cambiar" grafican y condensan la descripción de la escuela que realiza el directivo y operan como el suelo en el que se hace esta escuela. Las nociones recién citadas nos posibilitan allanar el camino para abordar en el siguiente apartado el trabajo que este director "bien ponderado/exitoso" desarrolla en la escuela seleccionada.

\section{EL DIRECTOR Y LA GESTIÓN DE LO COTIDIANO}

La NGP a nivel de las escuelas se materializa en un conjunto de creencias y de instrumentos asociados a las reformas del Estado y de su administración, vinculadas a políticas de achicamiento del Estado. Conjuntamente se promueven dinámicas participativas, que se constituyen en torno de la necesidad de refuerzo de la autonomía y de la responsabilización de las organizaciones del sector público. Asimismo se legitima una variante, más reciente, establecida en torno de una visión "calculadora y contable", desarrollada a partir de mecanismos de control a 
distancia, en los cuales es central el tratamiento de información por medio de dispositivos de vigilancia sobre el desempeño (CARVALHO, 2016). Estas dinámicas y principios en la escuela donde se realiza el trabajo de investigación se materializan principalmente en la delegación de mayores grados de responsabilidad al director sobre la obtención de recursos y la organización de procesos escolares cotidianos. Quizá por ello la figura del directivo es tan clave, y ser exitoso muchas veces demanda dedicarle la vida al cargo. Entre las acciones que le competen gestionar se destacan la planificación y ejecución del plan de mejora institucional, la capacitación de profesores, la formación en técnicas de gestión para la toma de decisiones, el uso de un sistema de información y monitoreo, el desarrollo de un Proyecto Educativo Institucional (PEI) y la realización de diversas rendiciones de cuenta a organismos ministeriales.

Organizamos este apartado a través de dos ejes que constituyen descriptores del trabajo cotidiano que el directivo desempeña: La mejora, una carrera de postas y Gestionar recursos: ¿̇crees en Papá Noel?

\subsection{La mejora, una carrera de postas}

Desde 2006 una de las principales acciones de política educativa han sido los Planes de Mejora Institucional (PMI). Estos Planes tienen como ámbito de definición y acción todas las escuelas secundarias del país. Según la matrícula de cada escuela, los PMI permiten asignar horas institucionales para el desarrollo de las actividades, además de un monto para los gastos operativos que dichas acciones requieran. El plan de mejora es la respuesta que desde las agencias oficiales se da para que las escuelas puedan poner en marcha un conjunto de acciones vinculadas a generar condiciones para garantizar el cumplimiento de la escolaridad obligatoria, demanda que la nueva legislación traía consigo. Así, a través de ese Plan el Estado genera condiciones para que cada escuela haga frente al pago de sueldos de docentes que trabajan fuera del horario de clases (tutorías extra escolares) y 
recursos para que las escuelas puedan organizar viajes y visitas, brindar viandas y refrigerios, adquirir materiales didácticos, herramientas, útiles, insumos informáticos, material audiovisual, entre otros. De este modo describe el director el proceso de recepción de estas políticas que se vienen se poniendo en marcha desde fines del siglo XX:

Primero fue PROMSE [Programa de Mejoramiento del Sistema Educativo], PROMEDU [Programa de Apoyo a la Política de Mejoramiento de la Equidad Educativa] y Plan de Mejora se llama ahora. Hay un referente de la Nación acá en Córdoba, hay varios mejor dicho, cada referente tiene un grupito de escuelas. Se llama Asistente Técnico Territorial (ATT). Entonces la escuela hace un proyecto, un plan de mejora y lo presenta al Asistente Técnico que es el que lo eleva a la Nación, previa revisión de la inspectora. Si es aprobado hay unos fondos que aporta Nación para gastos operativos, que tienen que tener relación con el plan, no puede ser cualquier tipo de gastos. No puede ser para tiza $\circ$ arreglar una cerradura, tiene que ver con el plan. $Y$ hay otro fondo que va asignado a los docentes tutores, nosotros tenemos 24 horas asignadas a los espacios de tutorías (ENTREVISTA DIRECTOR, AÑO 2015).

En el relato del director es posible identificar en primera instancia una historización de un conjunto de políticas educativas que en su registro son semejantes y que como señala han ido cambiando de nombre con los cambio de gobierno y de sus respectivos financiamientos: "plan de mejora viene de arrastre, derivación otros planes, PROMSE, PROMEDU (Entrevista director, año 2015)". La idea de "arrastre" supone reconocer que la lógica de la gestión por proyecto lleva años instaurada como una de las formas principales por las cuales el Estado gestiona las políticas educativas. Así, la racionalidad que la gestión por proyecto plantea a las escuelas se sostiene en el tiempo y les propone: planificar un proyecto, completar una extensa serie de formularios que deben ser aprobados por la inspección de escuela y finalmente recibir el "prometido" financiamiento.

De modo recurrente el director refiere al espacio de tutoría, que se desarrolla en contra turno escolar, como una de las acciones más destacadas de PMI dado los buenos resultados en materia de promoción 
escolar que viabiliza (AUTOR, 2015). A través del PMl la escuela financia las horas de tutoría que los docentes de la escuela brindan a los alumnos cuyo rendimiento escolar no es satisfactorio. Se trata de horas de trabajo por las que los docentes perciben un salario-extra sólo si ese financiamiento llega a la escuela. De otro modo, como reza la pregunta que da origen a este artículo, deben trabajar sin fondos.

Los alumnos pueden venir en el contraturno, sin gastos para ellos. En este espacio ellos hacen la tarea, completan la carpeta. Hay algunas situación familiar que se repite, hay mucho hacinamiento, entonces tenemos la biblioteca abierta mañana y tarde, hay una persona que está en la biblioteca siempre y si no lo cubrimos y el alumno está ahí, le prestamos la notebook. Así empezó desde que tenemos Plan de Mejora. Tenemos alumnos temporarios y hemos detectado algunos que les va bien y vienen lo mismo para no bajar la nota. Mientras no haya muchos no hay problema, el punto es que cuando se te juntan 20 ahí disminuye la calidad del servicio que se presta (ENTREVISTA DIRECTOR, AÑO 2015).

El espacio pedagógico de tutoría se presenta como una buena estrategia, "de calidad" para que el docente pueda trabajar con mayor detenimiento en las dificultades del alumno y elevar el nivel de promoción escolar. Ello claro está, cuando no ocurre, como señala el director, que este espacio optativo no se colme de alumnos.

Ahora bien, esta acción se articula, además, al Proyecto Educativo Institucional y al proyecto de gestión institucional que el director y el vicedirector deben diseñar. Esto no es un dato menor. La redacción en sí de cada proyecto implica horas y horas dedicadas a la cuestión. Es así como a la tarea de escribir y reescribir el PMl es referenciada en varias ocasiones como un proceso tedioso, que supone una gran inversión de tiempo, que es siempre escaso. Así lo describe:

El Plan de Mejora inicial fue reescrito varias veces, cambiamos varios asistentes. No nos poníamos de acuerdo con el Asistente Técnico Territorial (ATT), le pregunté qué quiere que ponga, cambié la hoja y listo. En la reunión con los directores, todos se quejaban del mismo ATT. Es profesor entonces no puede venir en los horarios que estamos disponibles. Entonces aparece y te pide montón de trabajo y 
después desaparece, eso enoja mucho. Veremos cómo terminamos. La verdad que no sé si es que pagan poco. Pero siempre se corta el proceso. Igual que cuando un curso cambia de profesor (ENTREVISTA DIRECTOR, AÑO 2015).

Como señala el director, no se trata de escribir un Plan que resulte de los debates y/o mejoras que la institución decide realizar, la escritura y reescritura aparece referenciada más bien como parte de algo tedioso que deben cumplir para complacer a un funcionario. Si algo de la elaboración de proyectos involucra la posibilidad del pensar y hacer con otros en la escuela, su formalización se vuelve más bien el cumplimiento tedioso de más carga burocrática para contar con la posibilidad de fondos extra que generalmente llegan a destiempo para atender al cumplimiento de la obligatoriedad escolar.

Así, lejos de volverse una herramienta ágil, la gestión por proyectos opera como un instrumento que incrementa exponencialmente el caudal de trabajo burocrático del director, promoviendo conductas de trabajo como "hacer lo que sea necesario sólo para recibir el aprobado" y finalmente esperar que los fondos lleguen, pagar el extra-sueldo a los docentes y poder cumplir con aquello que se reclama a las escuelas como compromiso y responsabilidad.

De modo que la mejora de la escolaridad pende del hilo de la reescritura de proyectos, la recepción de los fondos y de cambios de gobierno que pueden implicar tanto que ese plan se discontinúe, como modificaciones en los funcionarios ministeriales que llegan a la escuela con un nuevo "librito". De este modo refiere el director a la coyuntura política que atraviesa el Plan tras un cambio de gobierno:

Todo continúa pero no se sabe cómo. Tenemos que renovar un profe de Plan de Mejora, pero todavía no hemos podido empezar. Los chicos nos están preguntando por la tutoría. Cada dos o tres meses el profe cobraba, en marzo nos dijeron que no trabajen, pero en abril deberíamos arrancar (PRIMERA ENTREVISTA DIRECTOR, AÑO 2016). 
Los buenos resultados que el sistema de tutorías parece generar no alcanzan desde ya para garantizar su continuidad. Un programa atado a la gestión por proyectos se vuelve en la gestión del cotidiano escolar una carrera de postas para directivos, docentes y desde ya para los alumnos. La fragilidad de este tipo de programas genera que todo dependa de la voluntad del nuevo funcionario y de la agencia de directivos y docentes. Asimismo, genera en la escuela un vivir en permanente estado de incertidumbre: no saber cómo se sigue es un estado que la gestión por proyecto supone, particularmente cuando a través de sus recursos se financia el pago del trabajo que los docentes desarrollan. El peligro siempre latente es el cese en la renovación de los contratos de trabajo, es decir que el Plan desaparezca y junto con el la tutoría como un espacio pedagógico relevante para los alumnos. En el cotidiano escolar la contractualización (que en el caso de PMI supone recibir el salario cada dos o tres meses) como modalidad de intervención de la autoridad pública propia de la NGP (CARVALHO, 2016) transforma la ansiada sustentabilidad en gestión permanente de la incertidumbre. Como quien nunca llega a cumplir con los requerimientos, la gestión se vuelve una carrera de postas con final incierto y la precariedad de las condiciones laborales que este tipo de políticas instalan se transforma en moneda corriente.

\subsection{Gestionar recursos: ¿crees en Papá Noel?}

En Argentina el presupuesto educativo se destina principalmente al pago de salarios docentes. Esto supone que gestionar recursos para sostener el funcionamiento del edificio escolar léase ventanas, bancos, sanitarios, etc., se vuelva una responsabilidad de cada escuela y sus docentes sin, desde ya, contar con partidas presupuestarias para hacerlo. De esta forma lo describe el director:

El fondo de reparación el año pasado (año 2014) ninguna escuela lo cobró, hicimos notas con los directores y a final de año nos dieron un 
dinero. Son $250 \$ 66$ por mes, para colmo tengo que rendirlo en determinada cosa. Lo que yo hago es dejar que se acumule para rellenar los matafuegos, una parte la saco de ahí y otra por la cooperadora escolar, pero ni siquiera para eso. En el 2013 no se cobró nada directamente. Todo lo que se mantiene es por la cooperadora, estuvo intervenida por falta de transparencia en el manejo de los papeles. Yo me tuve que ocupar de hacer balances y la inspectora me notificó que habíamos hecho mérito para tener la cooperadora de vuelta (SEGUNDA ENTREVISTA DIRECTOR, AÑO 2015).

Aparece en el relato del director la cooperadora escolar como un agente central en el financiamiento de la escuela. Cabe señalar que la cooperadora es una figura que involucra a padres voluntarios que destinan parte de su tiempo a recaudar y administrar fondos. La cooperadora realiza diversas actividades: ventas de comida, eventos con los cuales obtiene dinero y paga incluso una línea de teléfono celular, dado que el teléfono fijo que la provincia provee no habilita tales llamadas; paga internet, la pintura y las reparaciones cotidiana que posibilitan que el edificio escolar se mantenga en buenas condiciones. A pesar de ser un factor necesario para la obtención de recursos económicos, la cooperadora no deja de ser una responsabilidad y un "mérito" del director, quien se vuelve garante de la "transparencia financiera" de esta organización. Claro está que este trabajo es necesario dado que el dinero que reciben es siempre escaso:

Y de pmi nos dan $5000 \$ 67$ que tampoco es mucho, hace tres años era eso y sigue igual, al año pasado compré dos cajas de resmas de hojas, afiches, fibrones y nada más (SEGUNDA ENTREVISTA DIRECTOR, AÑO 2015).

Es aquí donde la pregunta que lleva por título el presente artículo adquiere especial valor. Se cuestiona al director respecto de si sólo va a trabajar si recibe los fondos en medio del mar de agotamiento que la gestión de proyectos y sus tediosos procesos de rendición de cuentas involucran. Ello se vuelve más delicado y cruel si se presta atención a los irrisorios montos de dinero que las escuelas reciben tras cumplir con todo este trabajo. De hecho

\footnotetext{
66 Equivale aproximadamente al monto de 15 dólares.

${ }^{67}$ Equivale aproximadamente al monto de 314 dólares.
} 
la crueldad que la pregunta de este funcionario no es en vano. Los principios de la NGP están consiguiendo performar el trabajo del director tornándolo responsable de los procesos educativos y de sus resultados, más allá de los recursos económicos recibidos. En suma, esto supone trabajar sin los fondos. La lógica de la gestión por proyecto ha conseguido poner a directivos y docentes a trabajar y pensar de una determinada forma, gestionando recursos. Recuperando la pregunta del funcionario es factible afirmar que ese interrogante explicita por sí mismo algo que quienes transitamos por las escuelas de gestión estatal sabemos: si las escuelas funcionan es por el trabajo que hace el cuerpo directivo y docente. De forma que creer que las escuelas dependen del dinero de un plan, programa o proyecto para trabajar, o bien pensar que el director se sienta a esperar la llegada de los recursos es cómo creer en Papá Noel.

\section{ALGUNAS NOTAS CONCLUSIVAS}

Van Zanten (2012) explica que el aumento del caudal de trabajo concierne a todos los directores, sin embargo, es un problema que afecta cada vez a más a aquellos que trabajan en colegios e institutos "de la periferia de las grandes ciudades" donde asisten alumnos en desventaja socio-educativa. Esto se traduce en una multiplicidad de funciones que han de asumir los directores para evitar la implosión o relegación de su escuela, procurando promover y mantener una imagen como escuela, así como dar respuestas a las tensiones que conlleva la dificultad de conciliar la adaptación a las condiciones locales y el cumplimiento de las normas nacionales (AUTOR, 2015). El agobio por la cantidad de tareas a las que debe dar respuesta este director es una muestra de ello.

El trabajo extra, la recarga de tareas son componentes cotidianos del trabajo que el director debe hacer para poder responder a las demandas administrativas que la gestión por proyecto supone. Todo indica que las estrategias las debe generar cada escuela, conforme a los recursos que 
tiene, a los que proveen las políticas y a los que puedan autogestionar. Así, el director y los docentes se constituyen en expertos en "buscarle la vuelta" a las situaciones adversas que forman parte del día a día escolar en esta escuela de la periferia (AUTOR, 2011, 2015). La sobrecarga de trabajo se traduce en variados efectos como el cansancio o el stress. En términos de Ball (2002) las políticas también poseen "efectos de segundo orden" definidos por el impacto de las reformas educativas en el trabajo docente, cuestiones que involucran el gobierno de las emociones. Para los directivos esto se traduce en necesidad de asistencia psicológica, en enfermedades y malestar físico, entre otros efectos. Los paliativos para un trabajo que poco tiene que ver con los horarios formales son "obligarse" a disponer de tiempos para ocio y recreación, apagar el celular, no abrir el email en horario extra laboral, entre otras estrategias.

Gestionar una escuela estatal, con pocos recursos (o con recursos similares a los que reciben todas las escuelas de gestión estatal) y obtener buenos resultados en materia de escolarización obligatoria coloca al director en una posición de "modelo de gestor", en agente de "buenas prácticas de gestión". Ante este panorama, la vida escolar se dirime entre nuevos modos de gerenciamiento que apelan a legitimar una performance empresarial en el trabajo del director y una regulación de tipo moral que afecta y construye un nuevo ser-directivo. Ello acompañado por el discurso de la inclusión educativa, discurso que se materializa en frases recurrentes del director, tales como: "Si, no reciben esta oportunidad en la escuela, no la van a tener en ningún lado; por mis alumnos lo hago".

Consideramos que estamos asistiendo a la "producción en serie" de este tipo de directores que se vuelven "modelos" emblemáticos de la gestión eficaz y son quienes generalmente salen en diarios y noticieros. El componente moral que define el trabajo de este director "exitoso" se articula a procesos de afección (affect) que estas políticas instalan en el serdirectivo, configurando un nuevo deber-ser que redefine el trabajo que se debe promover para conducir una escuela. Así, la ética y el compromiso 
social adquieren centralidad en el discurso que justifica el trabajo que deben desarrollar.

La NGP involucra la regulación moral del "ser-directivo" al tiempo que apela a una ética propia de la autoayuda (AUTOR, 2008). La gestión de lo cotidiano se vuelve así un problema moral e individual donde la autogestión genera las condiciones para garantizar la escolarización secundaria obligatoria. Establecer la obligatoriedad de la enseñanza así es por lo menos barato, y sólo demanda volver el compromiso político y financiero para con la educación pública una cuestión moral e individual. Generalmente decimos que los procesos de regulación de las políticas educativas son complejos, sería importante añadir que en muchas ocasiones también son crueles.

\section{BIBLIOGRAFÍA}

AHMED, S. The promise of happiness. Durham and London: Duke University Press, 2010.

ALVARIÑO, C. Gestión escolar: un estado del arte de la literatura. Revista Paideia, n. 29, p. 15-43, 2000.

APPLE, M. W. Comparing neo-liberal projects and inequality in education. Comparative education, v. 37, n.4, p. 409-423, 2001.

BALL, S.; VAN ZANTEN, A. Logiques de marché et éthiques contextualisées dans les systèmes français et britannique. Éducation et sociétés, v.1, n. 1, p. 4771, 1998.

BALL, S. Reformar escolas/reformar professores e os terrores da performatividade. Revista Portuguesa de Educação, v.15, n. 2, p. 3-23, 2002.

BALL, S.; HOSKINS, K.; MAGUIRE, M.; BRAUN, A.; PERRYMAN, J. How schools do Policy. Policy enactments in secondary schools. New York: Routledge, 2012.

BALL, S. The education debate. London: Policy Press, 2013

BARRIGA, A.; ESPINOSA, C. I. El docente en las reformas educativas: Sujeto o ejecutor de proyectos ajenos. Revista lberoamericana de Educación, v. 25, p. 17-42, 2001.

BARROSO, J. Autonomia e Gestão das Escolas. Lisboa: Ministério da Educação, 1997. 
BARROSO, J. Politicas educativas e organização escolar. Lisboa: Universidade Aberta, 2005.

BERLANT, L. Cruel optimism. Durham and London: Duke University Press, 2011

CLARKE, J.; NEWMAN, J. The Managerial State. London: Sage, 1997

CARVALHO, L. M. Políticas educativas e governação das escolas. In: VI CICLO DE SEMINÁRIOS EM ADMINISTRAÇÃO, SUPERVISÃO E ORGANIZAÇÃO ESCOLAR, 2016, Porto. Anais Faculdade de Educação e Psicologia da Universidade Católica Portuguesa do Porto, 2016.

CASTRO GÓMEZ, S. Historia de la gubernamentalidad. Bogotá: Siglo del Hombre Editores, Pontificia Universidad Javeriana, Universidad Santo Tomas, 2010

DE MARINIS, P. Gobierno, gubernamentalidad, Foucault y los anglo foucaultianos. (Un ensayo sobre la racionalidad política del neoliberalismo). In: GARCÍA SELGAS, F. Y RAMOS TORRE, R. (Comp.). Retos actuales de la teoría social: globalidad, reflexividad y riesgo. Madrid: Centro de Investigaciones Sociológicas, 1999, p. 83-115.

DEAN, M. Governamentality. Power and rule in modern society. Londres: Sage Publications, 1999.

DELEUZE, G. El poder curso sobre Foucualt.Cactus: Buenos Aires, 2014

EACOTT, S. Liberating schools through devolution: the Trojan horse of the state. Leading and Managing, v. 17, n.1, P. 75-95, 2011.

ELMORE, R. F.; ABELMANN, C. H.; FUHRMAN S. H. "The New Accountability in State Education Reform: From Process to Performance." In: LADD H. F. (ed.), Holding Schools Accountable: Performance-Based Reform in Education. Washington D.C.: Brookings Institution, p. 23-63, 1996.

FOUCAULT, M. The archaeology of knowledge, trans. Alan Sheridan. London: Tavistock, 1972.

FOUCAULT, M. Nacimiento de la biopolitica. Buenos Aires: Fondo de la Cultura Económica, 1999.

FOUCAULT, M. Seguridad, territorio, población. Fondo de la Cultura Económica. Buenos Aires, 2006.

FOUCAULT, M. Nacimiento de la medicina social. In: FOUCAULT, M. Obras esenciales (pp. 653-671). Barcelona: Paidós, 2010.

HOOD, C. A public management for all seasons? Public Administration, v. 69, n. 1, p. 3-19,1991.

HODGSON, D. What do you want to do your life? Ethics and compulsory education. Journal of Education Policy, v. 31, n. 4, p. 495-507, 2016.

LUENGO, J.; SAURA, G. Mecanismos endógenos de privatización encubierta en la escuela pública. Políticas educativas de gestión de resultados y 
rendición de cuentas en Andalucía. Profesorado. Revista de currículum y formación del profesorado, v.16, n.3, p.111-126, 2012.

MAROY, C.; DUPRIEZ, $V$. La régulation dans les systèmes scolaires: proposition théorique et analyse du cadre structurel en Belgique francophone. Revue Française de Pédagogie: p.73-87, 2000.

MAROY, C. (Em direção a uma regulação pós burocrática dos sistema de ensino na Europa? In: ANDRADE OLIVEIRA D.; DUARTE, A. Políticas Públicas e Educação: regulação e conhecimento. Belo Horizonte: Fino Traço, p. 1946,2011.

MOORE, A.; CLARKE, M. 'Cruel optimism': teacher attachment to professionalism in an era of performativity. Journal of Education Policy, v.31, n.5, p.1-12, 2016.

POPKEWITZ, T. Sociología política de las reformas educativas. Madrid: Morata, 1994.

ROCKWELL, E. El trabajo docente hoy: Nuevas huellas, bardas y veredas. In: XI Congreso Nacional de Investigación Educativa, 2013, Distrito Federal. Anais Conferencias Magistrales. Consejo de Investigación Mexicano, 2013, p. 437473.

ROCKWELL, E. La experiencia etnográfica. Historia y cultura en los procesos educativos. Bs. As: Paidós, 2011.

ROSE, N. Powers of freedom. Reframing political thought. Reino Unido: Cambridge University Press, 1999.

ROSE, N.; O'MALLEY, P.; VALVERDE, M. " Governmentality". Revista Law \& Society, Annual Review, v. 2, n. 8, 2006.

SOTO LAGOS, R.; MERA ADASME, J.; NUÑEZ MUÑOZ, C.; SISTO CAMPOS, V.; FARDELLA CISTERNAS, C. Entre la efectividad y los afectos: nuevos docentes en tiempos de nuevo management público. Athenea Digital, v.16, n.3, p. 319, 2016. http://dx.doi.org/10.5565/rev/athenea.1528

VAN ZANTEN, A. L'école de la périphérie: Scolarité et ségrégation en banlieve (2e ed). Paris: PUF coll. Quadrige, 2012.

VIÑAO FRAGO, A. La cultura de las reforma escolares. Perspectivas Docentes, n.26, p. 38-56, 2002.

WHITTY, G.; POWER, S. AND HALPIN, D. La escuela, el estado y el mercado. Delegación de poderes y elección en educación, Madrid: Editorial Morata, 1998.

WILLMOTT, H. Strength is Ignorance; Slavery is freedom: Managing Culture in Modern Organizations. Journal of Management Studies, v.30, n.4, p. 215-252, 1993. 
WILKINS, A. Professionalizing school governance: the disciplinary effects of school autonomy and inspection on the changing role of school governors, Journal of Education Policy, v.30, n.2, p. 182-200, 2015.

Recebido em: Fevereiro de 2017 Aceito em: Abril de 2017 